\title{
PRUSSE
}

\section{LE $D^{r}$ ERNEST GURLT ${ }^{1}$}

Le 8 janvier dernier est décédé à Berlin, après une courte maladie, le $D^{r}$ Ernest Gurlt, professeur de chirurgie à l'Université de Berlin, un des membres les plus anciens et les plus respectés du Comité central allemand. Convaincu de la nécessité d'une organisation complète de l'assistance volontaire, il prit une part active à la fondation des Sociêtés allemandes de la Croix-Rouge. Il fut, en particulier, pendant de longues années, rédacteur du Kriegerheil.

Rappelons, à cette occasion, les détails biographiques que nous avons donné sur lui dans l'article que nous consacrions, en 1874, à son ouvrage paru en 1873 : Contribution à l'histoire de l'assistance volontaire et internationale des mulades en temps de guerre, et auquel, pour le surplus, nous renvoyons nos lecteurs ${ }^{2}$.

" Dès 1862, le $\mathrm{D}^{\mathrm{r}}$ Gurlt fut professeur de chirurgie à l'Université de Berlin. En 1864, il fut envoyé par le Comité central prussien, qui venait de se former, en Schleswig, sur le théâtre de la guerre, pour $\mathrm{y}$ faire des expériences personnelles relativement aux secours volontaires. Le résultat de ses observations, pendant les six semaines qu'il y passa, fut consigné dans un travail lu au Comité central et qui parut ensuite sous le titre de Fragments de chirurgie militaire. Au commencement de la guerre de 1866, il fut élu membre du Comité central et chargé comme tel, pendant les premières semaines, de l'organisation du dépôt central. Après la bataille de Langensalza, il conduisit le premier convoi de secours, et prit la direction d'un lazaret. Plus tard, il fut occupé en qualité de chirurgien consultant, dans les lazarets de Jüterbock, et dirigea ensuite le lazaret de la caserne des uhlans de la garde de Moabit, près Berlin. En 1867, il se renđit à Paris, comme délégué du Comité central prussien à l'exposition spéciale des Sociétés de secours et à la conférence préparatoire de la première Conférence internationale, qui dura de mars à juillet. En 1868, parurent ses

1 D'après Das Rothe Kreuz, 1899, $\mathrm{n}^{\circ} 3$.

2 Voy. T. V, p. 129 et T. X, p. 78. 
Planches descriptives du matériel des ambulances, avec texte allemand et français, dont il avait préparé les dessins à Paris. Il prit part comme secrétaire à la deuxième Conférence internationale tenue à Berlin en 1869. Au commencement de la guerre de 1870, il fut directeur du dépôt central, puis il partit comme chirurgien consultant pour les provinces rhénanes où il resta jusqu'en février 1871. Revenu à Berlin, à cette époque, il y remplit les mèmes fonctions dans une partie des lazarets du Tempelhof. »

Les ouvrages que nous connaissons de lui sont: Militärchirurgische Fragmente (Beriin, 1864); Denkschrift zur Beantwortung derjenige Fragen welche dem Professor Gurlt bei seiner Absendung nach dem Kriegschauplatze in Schleswig-Holstein ertheilten Instruction aufgestellt worden sind (Berlin, 1864); Abbildungen zur Krankenpflege im Felde auf Grund der internationalen Ausstellung zu Paris im Jahr 1866 (Berlin, 1868, atlas); La protection internationale des militaires blessés et malades en campagne, et l'assistauce volontaire en cas de guerre dans le royaume de Prusse (Berlin, 1869); Die freiwilligen Leistungen der Preussischen Nation in den Kriegsjahren 1813-1815. National Denkmal, (Berlin, 1872); Zur Geschichte der internationalen und freiwilligen Krankenpflege im Kriege (Leipzig, 1873); Neue Beiträge zur Geschichte der internationalen Krankenpflege im Kriege (Berlin, 1879).

L'an dernier, à l'occasion du jubilé cinquantenaire de son doctorat, sa réputation de chirurgien et de publiciste se manifesta par des témoignages de sympathie venus de toutes parts.

Sa disparition est une perte sensible pour le Comité central allemand ; son nom restera en honneur comme celui d'un collaborateur précieux dans les temps difficiles, ainsi que d'un zélé promoteur de tout ce qui pouvait favoriser le développement de l'assistance aux blessés.

\section{RUSSIE}

\section{LA GROIX-ROUGE PENDANT LA FAMINE ${ }^{1}$}

La Croix-Rouge russe a publié un compte rendu des secours organisés par elle lors de la famine occasionnée dernièrement par

' D'après Das Rothe Kreuz, 1899, $\mathrm{n}^{\circ} 3$. 